\title{
A brief introduction to climate change and health
}

To the Editor,

Our climate is changing at an unprecedented rate. This not only has impacts on the planet and the ecosystem, with extinction rates rising rapidly, but also on human health (Figure 1). The effects of climate change are and will continue to disrupt the basic requirements for health such as clean water, clean air, adequate food and will furthermore exacerbate underlying social, economic and ecological factors that cause illness and premature death. The repercussions of climate change should not be considered in isolation but also other factors of environmental change such as pollution, biodiversity loss and a resulting domino effect from species extinction, pesticide use and land use change need to be included.

Extreme weather events such as droughts, floods, landslides, wildfires and hurricanes all have severe impacts on human health. Injuries sustained from all the events pose a great risk alongside hazards of insufficient or contaminated water supplies, reduced food production leading to undernutrition, and cardiovascular and respiratory illnesses in the case of wildfires. Prolonged exposure to heat itself results in health impacts and excess mortality, such as heat stroke. It also has negative effects on the performance and productivity of individuals and their community as well as the economy and has been shown to lead to an increase in deaths from injury especially in youngto middle-aged men. Furthermore, it is important to consider that all extreme weather events also carry the threat of mental health issues, which also have long lasting effects to those afflicted.

Temperature extremes are increasing under climate change, and heat exposure was found to be associated with increased risk of cardiovascular, cerebrovascular and respiratory mortality. Furthermore, climate change is not only associated with an increase of temperature, but also with changes of the meteorological conditions and the chemical environment. Furthermore, there is evidence of synergistic effects between temperature and air pollution. Extreme weather events such as severe thunderstorms have also been shown to exacerbate asthma attacks, ${ }^{1}$ and climate change could also locally lead to an increase in the frequency of thunderstorms. Pollution as a concomitant factor can lead to worsening of asthma and other respiratory conditions. Wildfires such as the recent bushfires in Australia not only lead to immediate fatalities from the fires themselves but the resulting bushfire smoke also has severe effects on human health. To add a level of perspective, the PM2.5 levels were four times higher than the WHO guidelines in Sydney in December 2019. This trend of frequent and large bushfires will increase with climate change. ${ }^{2}$
Besides, climate, weather and environmental conditions have an indirect impact on allergies by influencing the levels, allergenic potential and types of pollen present. An overall increase in temperature will prolong the pollen season and can also lead to new sources of pollen due to new invasive species. Pollution enhances the susceptibility towards atopic diseases, for example by skin barrier disruption ${ }^{3}$ and exacerbates the symptoms of allergies. ${ }^{4}$ Pollution and climate change scenarios such as drought, high $\mathrm{CO}_{2}$, can also have a direct effect on the allergenicity of pollen ${ }^{5}$ (and other references).

Indirect effects of climate change have and will continue to affect infectious diseases. Changes in climate variables can alter the survival, reproduction or distribution of pathogens and hosts, as well as the availability and means of their transmission environment. However, the extent of these effects will vary depending on the disease and the location. In the case of malaria, modelling has shown that the overall malaria burden will increase due to climate change, and urbanization, migration, international trade and travel will further amplify disease incidence. ${ }^{6}$ Other infectious diseases such as dengue fever, Lyme disease, Zika, Chikungunya and even the recent SARS-CoV-2 outbreak are also examples of diseases where climate change will lead to an increased burden in health care.

Climate change and physical health issues have been discussed far more widely than the mental health effects, ${ }^{7}$ yet these also play an important role. The impact of extreme weather events and natural disasters can lead to mental health conditions such as post-traumatic stress disorder as well as conditions such as anxiety and have far more longer lasting effects. However, not only exposure to extreme weather events can lead to mental health problems. Distress caused by environmental changes, our knowledge there of and fear of an uncertain future itself, can lead to anxiety even without coming into direct contact with extreme weather events.?

As a means to counteract the effects of climate change, it is important to consider adaptation and resilience of the human body and of society. Additionally, it is essential to include social and economic factors when addressing the changing risk in the context of climate change. Some populations and areas will be able to respond more effectively to the stresses of climate change than others. These adaptations to climate change can themselves be classified into behavioural, infrastructure and technological adaptations ${ }^{6,8}$ which all can help to reduce the health impacts of climate change.

One essential adaptation that needs to be addressed is that the curricula for medical staff and, in particular, medical students need 
FIGURE 1 Climate change and the effects of human health. Adapted from: Helmholtz-Zentrum München-German Research Center for Environmental Health; from references ${ }^{9}$; Umweltbundesamt [German Federal Environment Agency], Klimawandel und Gesundheit [Climate change and health]. Infographic: Helmholtz (Icons:Shutterstock/Antun Hirsman, Flaticon)

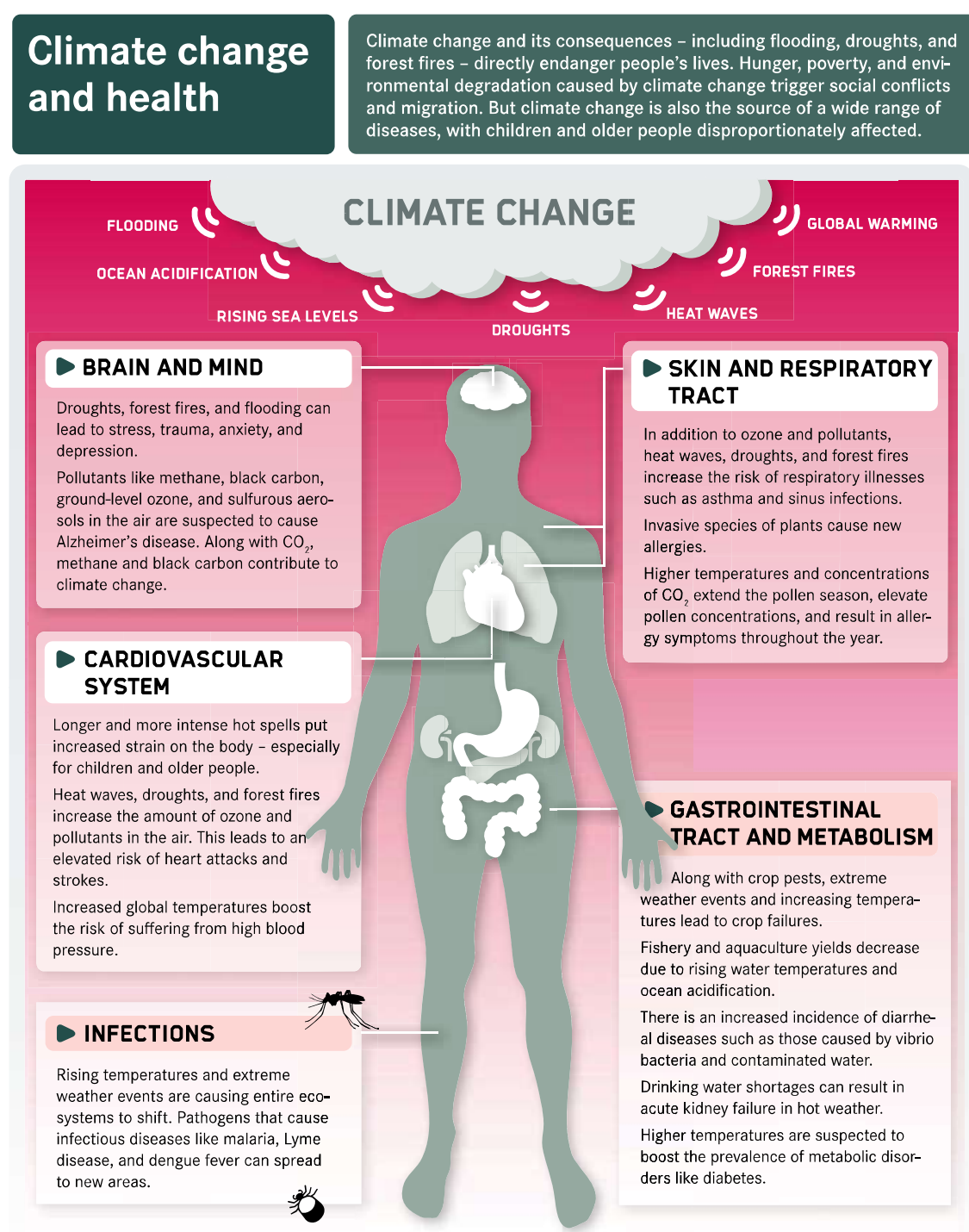

Source: Helmholtz-Zentrum München - German Research Center for Environmental Health; Watts N. et al., The Lancet 2019, doi.org/10.1016/50 140-6736(19)32596-6; Chen K. et al European Heart Journal 2019, doi.org/10.1093/eurheartj/ehz116; Umweltbundesamt [German Federal Environment Agencyl Klimawandel und Gesundheit [Climate K. et al., European Heart Journal 2019, doi.org/10.1093/eurheartj/ehz116; Umweltbund
change and health]. Infographic: Helmholtz (lcons: Shutterstock/Antun Hirsman, Flaticon) to include climate change and its effects on health. The health sector, responsible for $4.6 \%$ of the worldwide $\mathrm{CO}_{2}$ emissions, ${ }^{9}$ will itself need to address its overall emissions. Furthermore, the production, the supply chain and storage of essential drugs need to geographically diverse to minimize the impact on health care during and after extreme weather events.

In conclusion, the health benefits in mitigating the effects of climate change are obvious. A decrease in severe weather events, a stagnation of the increase of infectious diseases, and reductions of cardiovascular and respiratory stresses from heat or pollution would lead to an improvement of health in our daily lives. Additionally, choosing more environmentally friendly and hence often more active lifestyles is highly beneficial not only for the planet but also for our own health. The two main arguments for such measures are a disburdening of the healthcare system as well as afar better life quality for the affected people.
To ignore the effects of anthropogenic climate change would hence not only be disastrous for the planet and the environment but also have wide-ranging and complex problems for human health.

\section{KEYWORDS}

food allergy, infections, prevention, quality of life

\section{CONFLICT OF INTEREST}

Dr Hertig reports grants and personal fees from German Research Foundation, during the conduct of the study. Dr Traidl-Hoffmann reports grants and personal fees from Töpfer, personal fees from Sanofi, personal fees from Lilly, grants and personal fees from Sebapharma, personal fees from La Roche Posay, personal fees from Lancome and personal fees from Novartis, during the conduct of the study. Dr Fairweather has nothing to disclose. 


\section{Victoria Fairweather ${ }^{1,2}$ \\ Elke Hertig $^{3}$ \\ Claudia Traidl-Hoffmann ${ }^{1,2,4,5}$ (iD}

${ }^{1}$ Chair and Institute of Environmental Medicine, UNIKA-T,

Technical University of Munich, Munich, Germany

${ }^{2}$ Helmholtz ZentrumMünchen - German Research Center for

Environmental Health, Augsburg, Germany

${ }^{3}$ Faculty of Medicine, University of Augsburg, Augsburg,

Germany

${ }^{4}$ CK-CARE, Christine Kühne Center for Allergy Research and

Education, Davos, Switzerland

${ }^{5}$ Outpatient clinic for Environmental Medicine, University

Hospital Augsburg, Augsburg, Germany

Correspondence

Claudia Traidl-Hoffmann, Chair and Institute of Environmental Medicine, UNIKA-T, Technical University of Munich, Munich, Germany.

Email: claudia.traidl-hoffmann@tum.de

\section{ORCID}

Claudia Traidl-Hoffmann (D) https://orcid.

org/0000-0001-5085-5179

\section{REFERENCES}

1. D'Amato G, Annesi-Maesano I, Cecchi L, D'Amato M. Latest news on relationship between thunderstorms and respiratory allergy, severe asthma, and deaths for asthma. Allergy 2019;74(1):9-11.

2. Yu P, Xu R, Abramson MJ, Li S, Guo Y. Bushfires in Australia: a serious health emergency under climate change. Lancet Planet Health. 2020;4(1):e7-e8.

3. Heuson C, Traidl-Hoffmann C. The significance of climate and environment protection for health under special consideration of skin barrier damages and allergic sequelae. BundesgesundheitsblattGesundheitsforschung-Gesundheitsschutz. 2018;61(6):684-696.

4. Eguiluz-Gracia I, Mathioudakis AG, Bartel S, et al. The need for clean air: the way air pollution and climate change affect allergic rhinitis and asthma. Allergy. 2020;75:2170-2184. http://dx.doi.org/10.1111/ all.14177

5. Helander ML, Savolainen J, Ahlholm J. Effects of air pollution and other environmental factors on birch pollen. Allergy 1997;52:1207-1214.

6. Hertig E. Distribution of Anopheles vectors and potential malaria transmission stability in Europe and the Mediterranean area under future climate change. Parasit Vectors. 2019;12:18.

7. Usher K, Durkin J, Bhullar N. Eco-anxiety: How thinking about climatechange-related environmental decline is affecting our mental health. Int J Ment Health Nurs. 2019;28(6):1233-1234.

8. Hondula DM, Balling RC, Vanos JK, Georgescu M. Rising temperatures, human health, and the role of adaptation. Curr Clim Change Rep. 2015;1(3):144-154.

9. Watts N, Amann M, Arnell N, et al. The 2019 report of The Lancet Countdown on health and climate change: ensuring that the health of a child born today is not defined by a changing climate. Lancet 2019;394(10211):1836-1878. 